\title{
What the eye doesn't see: drugs psychiatrists and GPs don't know their patients are on
}

\author{
Nicholas A. Clarke, Research Associate, Miriam Marks Department of \\ Neurochemistry, Institute of Neurology, London WC1N 1PJ and UMDS Guy's \\ Campus, London SE1 9RT
}

Personal experience shows that discrepancies are common when out-patient psychiatric medication records are checked with the information held by a patient's GP. This could lead to duplicated or conflicting treatment regimes, dangerous drug interactions, abuse of prescribed drugs, and failure to monitor therapeutic drug levels.

This study compares the information held by psychiatrists and GPs about psychiatric outpatients' consumption of prescribed and unprescribed medication; this information was used to examine the efficacy of communication about these drugs. Prasher et al (1992) showed that $68 \%$ of GPs listed medication on referral of 270 people to outpatient departments, while $95 \%$ of psychiatrists listed drugs in their reply-drug records were not compared.

\section{The study}

A single psychiatric out-patient clinic session for 20 patients was selected, at Barnet, Hertfordshire, a London suburb with a population of 200000 . The records were examined of 16 patients being seen at repeat appointments, of three being seen after rereferral 'de novo', and of one patient who did not attend. There were no completely new patients.

Out-patient and general practice medication records and letters were audited for the preceding six months.

Drug information from the hand-written outpatient notes, pharmacy cards (available for 14 of the patients), original GP letter (if any), and the copies of letters sent to the patient's GP were compared with GP medication records for the same patient. The latter items were obtained by telephone and in one case an additional letter to the patient's GP.

Every patient's GP or GP partner was contacted; medication details of ten patients were held by the GP on computer.

All references to prescribed or unprescribed drugs, with most recent dosage, and entries of 'no medication' were noted. Changes to medication that session were not included.

\section{Findings}

There were 11 women (aged 40-70 years) and nine men (aged 30-81 years). Twelve had recent principal diagnoses of depressive illness, four of anxiety neurosis and one each of alcohol dependence, schizophrenia, gender dysphoria and specific learning difficulties.

All 19 medicated patients had drug usage recorded in their notes and all had clinic letters to their GP. The unmedicated patient was recorded as such by both parties. One patient was excluded as the GP was unable to supply a medication history.

Drugs omitted by either one or both parties numbered 41, from a total number prescribed of 58 . Psychiatrists omitted 29 completely in both notes and letters. Their omissions from clinic letters totalled 35 and from written notes 32 , affecting 38 prescriptions for 12 patients.

It was not possible to ascertain how many drugs were omitted from all three records, as patients were not interviewed.

The psychiatrist's medication records for one patient omitted 16 out of 17 prescribed drugs. One of those omitted was a psychotropic drug (a benzodiazepine); the monoamine oxidase inhibitor (MAOI) was recorded.

Non-psychotropic medication dominated the psychiatrists' remaining omissions. Eleven other patients had six or fewer omissions, involving 22 different drugs of which only four were psychotropics (a serotonergic and a tricyclic antidepressant, a major tranquilliser and another benzodiazepine). Thus non-psychotropics represented 33 of 38 psychiatrists' omissions: aspirin, four NSAID (nonsteroidal anti-inflammatory drug) tablets and two NSAID gels, thyroxine, a $\mathrm{H}_{2}$ receptor antagonist, five paracetamol-based analgesics, an antihypertensive, a thiazide diuretic, an antiepileptic, diphenoxylate, four antibiotics, steroid and salbutamol inhalers, a smooth-muscle antispasmodic, folate, iron, two vitamin tablets, steroid cream, emollient cream, a stool softener, and an influenza vaccine.

GPs omitted ten out of 58 drugs, all known to the psychiatrists, involving eight patients, two subjects 
having two drugs omitted in their records. In contrast to the psychiatrists, six GPs omissions were of psychotropic drugs, comprising two serotonergic and a tricyclic antidepressant, a major tranquilliser and two benzodiazepines. The remaining four omissions were of aspirin and an NSAID tablet, a paracetamol-based analgesic, and iron tablets.

All agreed drugs between the GP and psychiatrists were psychotropic. These totalled 17 of the 58 drugs, involving 13 patients: two were lithium, one disulfiram, seven tricyclic and one serotonergic and one MAOI antidepressants, three major tranquillisers, a benzodiazepine and an anticholinergic drug were all agreed, the maximum for one patient being two drugs.

The ten subjects with computerised GP records included only two of the six cases with no omissions or dose discrepancy between the two parties.

\section{Comment}

Of great concern is the finding that, of 58 drug prescriptions, 41 were omitted from the records of either psychiatrist or GP, 33 of which were for non-psychotropic drugs.

Of the 17 drugs known to both parties there was still missing dosage information in ten.

Of the 33 omissions of non-psychotropic medications, psychiatrists missed 27 entirely, and the six remaining in either letters to the GP or in their own notes. This bias may reflect a tendency by both patients and doctors to favour attention to the area of illness (and therefore treatment) for which advice is being sought and for the psychiatrist to perhaps believe much non-psychotropic medication is of limited relevance to their practice.

An important potential adverse drug interaction is between lithium and NSAIDs/aspirin, and yet the prescription of NSAIDs/aspirin was omitted seven times, in the notes of four patients. Such drugs may also be purchased over the counter, unknown to either doctor. Other noteworthy interactions with lithium include diuretics, angiotensin-converting enzyme inhibitors and calcium channel blockers.

Only eight out of 25 psychotropic drugs which may reflect a mutually perceived area of special expertise in the clinic. Importantly, both lithium records were free of omissions, as is consistent with emphasis laid on its careful control.

Possible interventions might include the use of a 'shared care card' (Essex; 1990) or mailed postcards to patients reminding them to bring medication to a clinic (Penner; 1991) A larger study using patient interviews could include closer examination of drug recording in relation to clinic specialty and to patient compliance.

In the interests of patient safety, care should be taken by specialists to enquire about medication not obviously related to his or her area of expertise.

\section{Acknowledgements}

The author thanks Dr L. Sireling, consultant psychiatrist at Barnet Hospital, for much helpful advice and constructive criticism in drafting, and also the general practitioners and FHSAs of the area. This study was supported in part by a Priory Hospital Research Fellowship (Guy's Hospital).

\section{References}

Essex, B., Dolg, R. \& Renshaw, J. (1990) Pilot study of records of shared care for people with mental illnesses. British Medical Journal, 300, 1442-1446.

Penner, M., Dicker, M. \& EnS, S. (1991) Using postcards to increase patient provided information on medication use. Family Medicine, 23, 44-45.

Prasher, V. P., Fitzmaurice, D., Krishnan, V. H. R. \& OYEBODE, F. (1992) Communication between general practitioners and psychiatrists. Psychiatric Bulletin, 16, 468-469. 